\title{
A NOTE ON A THEOREM OF JACOBSON
}

\author{
T. S. RAVISANKAR
}

The question as to whether every derivation of a simple algebra is inner, is still unsettled. The simple proofs given below of Theorem A, which is a special case of a well-known theorem (see [4, pp. 22-23]), and that of Theorem B would possibly be a new approach to this question.

TheOREM A. If $A$ is a simple algebra with identity over an algebraically closed field $F$ of characteristic zero, then every derivation of $A$ is inner.

Proof. In the first instance, let $A$ have an identity or not. Let $R_{x}\left(L_{x}\right)$ denote the right (left) multiplication in $A$, and $D$ be a derivation of $A$. Then $A$ has neither proper ideals nor proper $D$-ideals. In other words, the Lie algebra $L\left(L^{\prime}\right)$ generated by $R_{x}, L_{x}\left(R_{x}, L_{x}\right.$ and the derivation $D$ ) is irreducible. Moreover, $L^{\prime}=L+\{\alpha D\}_{\alpha \in F}$ (a vector space sum and not necessarily a direct sum), and $L$ is an ideal of $L^{\prime}$. Further, by a theorem of Jacobson $[1$, p. 47], we have $L$ $=C \oplus[L L] ; L^{\prime}=C^{\prime} \oplus\left[L^{\prime} L^{\prime}\right]$ for centers $C, C^{\prime}$ of $L, L^{\prime} ;[L L],\left[L^{\prime} L^{\prime}\right]$ are semisimple; $[L L]$ is an ideal of $\left[L^{\prime} L^{\prime}\right]$. Any transformation $T$ in $C$ commutes with the irreducible associative algebra generated by $R_{x}, L_{x}$ and hence should be a multiple of the identity transformation $I$, by Schur's lemma. Now, if $A$ contains an identity, $C=F I, C^{\prime}=F I$; since the dimension of $L^{\prime}$ is at the most greater by unity than that of $L$, and since $\left[L^{\prime} L^{\prime}\right]$ cannot have a one dimensional (abelian) ideal complementary to $[L L],[L L]=\left[L^{\prime} L^{\prime}\right]$; i.e., $L=L^{\prime}$, or, $D \in L$. Thus, every derivation of $A$ is inner.

REMARK 1 . In case $A$ is any simple nonassociative algebra, then $C \subseteq C^{\prime}$ and therefore $[L L]=\left[L^{\prime} L^{\prime}\right]$ in this case also. If, in addition $C=C^{\prime}$ for every derivation $D$ of $A$, then every derivation of $A$ will be inner. Because $F$ is algebraically closed, $C=0$ or $C=F I, C^{\prime}=0$ or $C^{\prime}=F I$. Since $C=F I$ implies $C^{\prime}=F I=C$ and since every derivation is inner in this case as well as when $C^{\prime}=0$, the question raised at the outset boils down to the consideration of the only case $C=0, C^{\prime}=F I$. The plausibility of this case remains to be seen.

Now, in the case of simple Lie algebra $A$ over a field $F$ of characteristic zero, $L=\{\operatorname{ad} x\}_{x \in A} ; L^{\prime}=L+\{\alpha D\}_{\alpha \in \boldsymbol{F}}$. Since $A$ is simple, the center of $A=\{x \in A \mid$ ad $x=0\}=\{0\}$. If ad $y \in \operatorname{center} C$ of $L$, then

Received by the editors August 20, 1968. 
$[\operatorname{ad} x, \operatorname{ad} y]=\operatorname{ad}[x, y]=0$ for every $x$ in $A$; hence $[x, y]=0$, i.e., ad $y=0$ and $C=0$. If ad $z+\alpha D$ belongs to the center $C^{\prime}$ of $L^{\prime}$, then $[\operatorname{ad} z+\alpha D, \operatorname{ad} x]=0$ for all $x$, i.e., ad $[z, x]-\alpha$ ad $x D=0$ for all $x$ in $A$, or, $[z, x]-\alpha x D=0$, i.e., $-(\operatorname{ad} z+\alpha D)=0$. Hence $C^{\prime}=0$. Thus $L, L^{\prime}$ are semisimple $[1$, p. 47]. Further arguments as in the proof of Theorem A yield

TheOREM B. Every derivation of a simple Lie algebra $A$ over a field of characteristic zero is inner.

REMARK 2. Theorem B is, more generally, true for a simple Malcev algebra $A$ [3]. For, the center $C$ of $L$ is then known to be the zero ideal [2, Corollary 5.32]. A similar argument shows that the center $C^{\prime}$ of $L^{\prime}$ is also the zero ideal.

\section{REFERENCES}

1. N. Jacobson, Lie algebras, Interscience, New York, 1962.

2. A. A. Sagle, Malcev algebras, Trans. Amer. Math. Soc. 101 (1961), 426-458.

3. - On derivations of semi-simple Malcev algebras, Portugal. Math. 21 (1962), 107-109.

4. R. D. Schafer, An introduction to nonassociative algebras, Academic Press, New York, 1966 .

The Ramanujan Institute, University of Madras 